Bisheng Du \& Christian Larsen

Base stock policies with degraded service to larger orders

CORAL - Centre for Operations

Research Applications in Logistics 


\title{
Base stock policies with degraded service to larger orders
}

\author{
Bisheng Du \\ Christian Larsen \\ CORAL $^{1}$, Department of Business Studies \\ Aarhus School of Business \\ University of Aarhus \\ DK-8210 Aarhus V \\ Denmark
}

\begin{abstract}
We study an inventory system controlled by a base stock policy assuming a compound renewal demand process. We extend the base stock policy by incorporating rules for degrading the service of larger orders. Two specific rules are considered, denoted Postpone $(q, t)$ and $\operatorname{Split}(q)$, respectively. The aim of using these rules is to achieve a given order fill rate of the regular orders (those of size less than or equal to the parameter $q$ ) having less inventory. We develop mathematical expressions for the performance measures order fill rate (of the regular orders) and average onhand inventory level. Based on numerical experiments, our conclusion is that $\operatorname{Split}(q)$ seems to perform best.

Keywords: Base stock policy, compound renewal process, order fill rate, differentiated service.
\end{abstract}

\section{Introduction}

It is well known that the larger demand variation is the higher inventory levels are needed in order to secure adequate service from an inventory system. One reason for large demand variation may be that the system occasionally receives large customer orders. Furthermore, these large orders may have negative effects further upstream in the supply chain. Therefore, as a manager of an inventory system, you would prefer to receive smaller orders at a more frequent pace rather than receiving orders with large variation at a less frequent pace. However, assuming that in the short-run you cannot do anything to change the order behaviour of your customers, it may be sensible to introduce a policy, implying degraded service to larger orders. Furthermore, customers submitting larger orders may very well be aware of the inconvenience that they cause and be willing to accept degraded service.

The considerations raised here are inspired by a discussion that the authors had recently with the logistics personnel in a larger Danish company where the second author has been involved in an inventory control project. The aim of this project was to decide optimal base stock levels when the service measure is order fill rate, that is, the fraction of orders received, where the whole order is delivered instantaneously from the inventory. This project was reported in Larsen et al. (2008) and some theoretical aspects of the project in Larsen and Thorstenson (2008). Obviously, large orders can have very negative effects on the order fill rate service measure (thus, using this service measure, it is implicitly assumed that smaller orders are just as important as larger ones).

As the source of inspiration is this company project, it is natural that the mathematical model developed in this paper takes its point of departure in the model of this project. Therefore, we study

\footnotetext{
${ }^{1}$ Centre for Operations Research Applications in Logistics, e-mail adressses: bisd@asb.dk and chl@asb.dk
} 
a base stock system with control parameter $S$ and where all replenishment orders, issued instantaneously upon receipt of an order, have a constant lead-time $L$. Our extension of the model is the introduction of a given positive integer $q$, based on which orders are distinguished as being either regular orders (of a size less than or equal to $q$ ) or large orders (of a size larger than $q$ ). We introduce two rules, $\operatorname{Split}(q)$ and Postpone $(q, t)$, which both seek to serve regular orders as well as possible while degrading the service of larger orders. Postpone $(q, t)$ operates as follows: the parameter $t$ is in the interval between 0 and $L$. When receiving a large order, say at time point $\bar{\tau}$, the order is deliberately backlogged for $t$ time units before it is attempted to be served. This implies that all regular orders arriving in the time interval $(\bar{\tau}, \bar{\tau}+t)$ are served ahead of this large order. Another consequence is that the effective lead-time of the larger orders is $L-t$. $\operatorname{Split}(q)$ operates as follows: each time a large order is received, it is split into a suborder of size $q$ and a suborder of the remaining size. The first suborder is then treated as a regular order while the second suborder is handled outside the inventory system, directly from the supply system, thus having a lead-time $L$. Therefore, the inventory system only faces orders that are less than or equal to $q$ and all replenishment orders are the original order eventually truncated by $q$. Which of the two rationing rules will be most accepted by the customers of the large orders? We believe that for a given $q$, there exists a threshold value of $t$ making the large customers indifferent between the two rules. Later in the paper, we provide a reasonable (and easy to compute) method for deciding this threshold value. The choice of which rule to apply is then based on which of the two gives the lowest average on-hand inventory level subject to the requirement that the regular orders have a given order fill rate.

There has been a large number of studies of inventory control in the presence of several (most often two) customer classes. For a good overview of the literature, see Teunter and Haneveld (2008). The aim of these studies is to design rationing rules concerning when to backlog (or reject) the demand of the least important customer class when the inventory level is critically low. The main distinction between our paper and this line of research is that we do not explicitly model several customer classes and we discriminate with respect to time and not quantity. We do consider discrimination, but this is in relation to delaying the service of certain orders (in our case the larger orders). Thus, we do not introduce any critical numbers on the inventory levels for when to deny service of some orders. Therefore, our work is not so closely related to the traditional studies of rationing policies in inventory control systems. When considering the rule Postpone $(q, t)$, our work is more in line with the paper of Wang et al. (2002) which is motivated by a company analysis reported in Cohen et al. (1999). They consider a base stock system with two customer classes where the service of one of the classes is first attempted after a given time period (in the paper denoted demand or delivery lead times) has elapsed since the receipt of the order. As the demand model therein is a Poisson process (thus without a compound element), the concern about degrading service of larger orders is obviously outside the scope of this paper. Furthermore, we also generalize the demand model by considering a renewal process instead of a Poisson process. As an aside, we note that recently a paper by Kocuga and Sen (2007) has been published which extends the model of Wang et al. (2002) by introducing critical number rules as seen in the rationing literature, featured in Teunter and Haneveld (2008). When considering the rule Split(q), our work has some relation to studies of order splitting and multiple sourcing. For a review of these studies, see Thomas and Tyworth (2006). However, as we only use $\operatorname{Split}(q)$ to be able to make comparisons with another rule, Postpone $(q, t)$, which cannot be cast into the framework of the splitting/sourcing literature, our work cannot be compared to results obtained in this field. 
In Section 2, we derive mathematical expressions for the order fill rate (of the regular orders) and the average on-hand inventory for the rules $\operatorname{Split}(q)$ and Postpone $(q, t)$. For each rule, this is done in two stages: first for a general compound renewal process and then in the case of a compound Erlang process, making the mathematical expressions more computable. We finish this section by deriving a reasonable threshold value of $t$ that we find will make customers of larger orders indifferent between the two rules. Then in Section 3, we present the results of a numerical study. Finally, we state some concluding remarks in Section 4.

\section{Mathematical model}

\subsection{Preliminaries}

The demand process is a compound renewal process where the time between order arrivals is specified by a positive continuous random variable $T$. The size of a customer order is specified by a positive integer valued random variable $X$. As stated in the previous section, we assume a given positive integer $q$ that distinguishes between being regular and large orders. We imagine that $q$ is reasonably large, for instance the $90 \%$ or the $95 \%$ quantile of $X$, as seen later in our numerical experiments.

Let the random variable $X^{R e g}$ denote the size of a regular order. It has probability distribution

$$
P\left(X^{\operatorname{Re} g}=x\right)=\frac{P(X=x)}{P(X \leq q)} \quad x=1,2, \ldots, q
$$

For any non-negative integer $m$, define the random variable $Q(m)$ as

$$
Q(m)=\sum_{i=1}^{m} X_{i}
$$

where $X_{i}, i=1, . ., m$ are independent and identically distributed as $X$. Per definition, $P(Q(0)=0)=1$.

Similarly, for any non-negative integer $m$, define the random variable $Q^{R e g}(m)$ as

$$
Q^{\operatorname{Re} g}(m)=\sum_{i=1}^{m} X_{i}^{\operatorname{Re} g}
$$

where $X_{i}^{\operatorname{Reg}}, i=1, . ., m$ are independent and identically distributed as $X^{\operatorname{Reg}}$. Per definition, $P\left(Q^{R e g}(0)=0\right)=1$.

For later use, we state

\section{Lemma 1}

When $x \leq q$, it holds that $P(Q(m)=x)=(P(X \leq q))^{m} P\left(Q^{\operatorname{Re} g}(m)=x\right)$

Proof: See Appendix. 
Let $\tau$ be an arbitrarily chosen time point which in our paper can either be a time point of an arrival of a customer with a regular order or a randomly chosen time point. For any non-negative real number $s$, the random variable $N(\tau)_{s}$ is the number of customer arrivals in the time interval $[\tau-s, \tau)$.

\subsection{Rule $\operatorname{Split}(q)$}

We first develop an expression for the order fill rate. Let $\bar{\tau}$ be the time point of an arrival of a customer with a regular order. Let the random variable $\bar{D}_{L}$ denote the aggregate demand recorded in the inventory system in the time interval $[\bar{\tau}-L, \bar{\tau}$ ). As all larger orders are truncated by $q$ (and the remaining order of any larger order is handled outside the inventory system), the probability distribution of $\bar{D}_{L}$ can be specified as follows.

When $x=0$ or $q=1$

$P\left(\bar{D}_{L}=x\right)=P\left(N(\bar{\tau})_{L}=x\right)$

and when $\mathrm{x}>0$ and $q>1$

$$
\begin{aligned}
& P\left(\bar{D}_{L}=x\right) \\
& =\sum_{m=\operatorname{Roof}(x / q)}^{x} P\left(N(\bar{\tau})_{L}=m\right) \sum_{y=\operatorname{Roof}((q m-x) /(q-1))}^{m}\left(\begin{array}{c}
m \\
y
\end{array}\right)(P(X \leq q))^{y}(P(X>q))^{m-y} P\left(Q^{r e g}(y)=x-(m-y) q\right)
\end{aligned}
$$

$\operatorname{Roof}(a)$ is the smallest integer greater than or equal to the real number $a$ and $\left(\begin{array}{l}m \\ y\end{array}\right)$ is the binomial coefficient. The order fill rate service measure, measuring the service given to regular orders and abbreviated $O F R$, is then

$$
\operatorname{OFR}_{\text {Split(q) }}(S)=P\left(X^{\operatorname{Reg}}+\bar{D}_{L} \leq S\right)
$$

We now develop expressions for the average on-hand inventory level. Let $\tilde{\tau}$ be a randomly chosen time point. Let the random variable $\tilde{D}_{L}$ denote the aggregate demand recorded in the inventory system in the time interval $[\tilde{\tau}-L, \tilde{\tau})$. Then, as above, the probability distribution of $\tilde{D}_{L}$ can be specified as follows.

When $x=0$ or $q=1$ :

$P\left(\tilde{D}_{L}=x\right)=P\left(N(\tilde{\tau})_{L}=x\right)$

and when $x>0$ and $q>1$

$$
P\left(\tilde{D}_{L}=x\right)
$$


$=\sum_{m=\operatorname{Roof}(x / q)}^{x} P\left(N(\tilde{\tau})_{L}=m\right) \sum_{y=\operatorname{Roof}((q m-x) /(q-1))}^{m}\left(\begin{array}{c}m \\ y\end{array}\right)(P(X \leq q))^{y}(P(X>q))^{m-y} P\left(Q^{r e g}(y)=x-(m-y) q\right)$

The average on-hand inventory level is

$I_{S p l i t(q)}(S)=\sum_{x=0}^{S-1} P\left(\tilde{D}_{L}=x\right)(S-x)$

For the case where $T$ is a $k$-phased Erlang distribution with mean $k / \lambda$ (that is, $\lambda$ is the intensity of the underlying Poisson process), we have (see for instance Cox (1962) p. 39)

$P\left(N(\bar{\tau})_{L}=n\right)=e^{-\lambda L} \sum_{j=n k}^{(n+1) k-1} \frac{(\lambda L)^{j}}{j !} \quad n=0,1,2, \ldots$

and

$P\left(N(\tilde{\tau})_{L}=n\right)=\left\{\begin{array}{cc}e^{-\lambda L} \sum_{j=0}^{k-1} \frac{k-j}{k} \frac{(\lambda L)^{j}}{j !} & n=0 \\ e^{-\lambda L} \sum_{j=1-k}^{k-1} \frac{k-|j|}{k} \frac{(\lambda L)^{j+n k}}{(j+n k) !} & n=1,2, \ldots\end{array}\right.$

One can thus get computable expressions in order to do numerical analysis of $\operatorname{Split}(q)$ for the case of a compound Erlang process.

\subsection{Rule Postpone $(q, t)$}

First, we derive a measure for the order fill rate. Let $\bar{\tau}$ be the time point of a customer arrival. Let the random variable $\bar{C}_{L}(t)$ denote what we define to be the committed aggregate demand in the time interval $[\bar{\tau}-L, \bar{\tau})$. This is all the recorded demand in the interval $[\bar{\tau}-L, \bar{\tau}-t)$ and all the recorded demand of the regular orders in the time interval $[\bar{\tau}-t, \bar{\tau})$. The reason why only demand of the regular orders is counted in the latter interval is that all larger orders received in this interval are still denied access to the inventory at time point $\bar{\tau}$. The probability distribution of $\bar{C}_{L}(t)$ can be specified as follows.

$P\left(\bar{C}_{L}(t)=0\right)=P\left(N(\bar{\tau})_{L}=0\right)+\sum_{m=1}^{\infty}(P(X>q))^{m} P\left(N(\bar{\tau})_{L}=m, N(\bar{\tau})_{t}=m\right)$

When $\mathrm{x}>0$ 


$$
\begin{aligned}
& P\left(\bar{C}_{L}(t)=x\right) \\
& =\sum_{m=1}^{\infty} \sum_{r=\max \{m-x, 0\}}^{m} P\left(N(\bar{\tau})_{L}=m, N(\bar{\tau})_{t}=r\right) \sum_{y=0}^{\min \{x-m+r, r\}}\left(\begin{array}{l}
r \\
y
\end{array}\right)(P(X \leq q))^{y}(P(X>q))^{r-y} P\left(Q(m-r)+Q^{\operatorname{Reg} g}(y)=x\right)
\end{aligned}
$$

The two random variables $N(\bar{\tau})_{L}$ and $N(\bar{\tau})_{t}$ are positively correlated and $P\left(N(\bar{\tau})_{L} \geq N(\bar{\tau})_{t}\right)=1$. The order fill rate service measure is

$$
\operatorname{OFR}_{\text {Postpone }(q, t)}(S)=P\left(X^{\text {reg }}+\bar{C}_{L}(t) \leq S\right)
$$

We now derive a measure for the average on-hand inventory level. Let $\tilde{\tau}$ be a randomly chosen time point. Let the random variable $\tilde{C}_{L}(t)$ denote the committed aggregate demand in the time interval $[\tilde{\tau}-L, \tilde{\tau})$. As previously defined, this is all the recorded demand in the interval $[\tilde{\tau}-L, \tilde{\tau}-t)$ and all the recorded demand of the regular orders in the time interval $[\tilde{\tau}-t, \tilde{\tau})$. The probability distribution of $\tilde{C}_{L}(t)$ can be specified as follows.

$$
P\left(\tilde{C}_{L}(t)=0\right)=P\left(N(\tilde{\tau})_{L}=0\right)+\sum_{m=1}^{\infty}(P(X>q))^{m} P\left(N(\tilde{\tau})_{L}=m, N(\tilde{\tau})_{t}=m\right)
$$

When $\mathrm{x}>0$

$$
\begin{aligned}
& P\left(\tilde{C}_{L}(t)=x\right) \\
& =\sum_{m=1}^{\infty} \sum_{r=\max \{m-x, 0\}}^{m} P\left(N(\tilde{\tau})_{L}=m, N(\tilde{\tau})_{t}=r\right) \sum_{y=0}^{\min \{x-m+r, r\}}\left(\begin{array}{l}
r \\
y
\end{array}\right)(P(X \leq q))^{y}(P(X>q))^{r-y} P\left(Q(m-u)+Q^{\operatorname{Reg}}(y)=x\right)
\end{aligned}
$$

There is a similar relationship between the two random variables $N(\tilde{\tau})_{L}$ and $N(\tilde{\tau})_{t}$ as between the random variables $N(\bar{\tau})_{L}$ and $N(\bar{\tau})_{t}$. The average on-hand inventory level is specified as

$$
I_{\text {Postpone }(q, t)}(S)=\sum_{x=0}^{S-1} P\left(\tilde{C}_{L}(t)=x\right)(S-x)
$$

A major problem in computing the expressions derived so far for Postpone $(q, t)$ is the presence of the joint probabilities $P\left(N(\bar{\tau})_{L}=m, N(\bar{\tau})_{t}=r\right)$ and $P\left(N(\tilde{\tau})_{L}=m, N(\tilde{\tau})_{t}=r\right)$. We now consider the case where $T$ is a $k$-phased Erlang distribution with mean $k / \lambda$. Note that a $k$-phased Erlang distribution can be subdivided into $k$ phases, each having a duration that is exponentially distributed with mean $1 / \lambda$. Each time a phase completes, we can interpret it as an "arrival", where it is only every $k$ th arrival that is real while the others are fictitious. Thus, the process will at any time point be in one of the phases $1,2, \ldots, k$. Being in phase $i$ means that $k-i$ fictitious arrivals have elapsed since the last real arrival, see Figure 1 (note that as we look backward in time when doing mathematical derivations, we also state the phase numbers accordingly). 
$<$ Figure 1 about here $>$

When we use the word arrival in the following, it should be understood as a real arrival. Let $\tau$ be an arbitrarily chosen time point. Denote by $F(i, r, t \mid j)$ the conditional probability that given that we are in phase $j$ at time point $\tau$, we are in phase $i(i=1,2 .,, k)$ at time point $\tau$ - $t$ and the total number of arrivals in the time interval $[\tau-t, \tau)$ is $r$. Then

$F(i, r, t \mid j)=e^{-\lambda t} \frac{(\lambda t)^{r k+i-j}}{(r k+i-j) !} \quad i=1, . ., k ; j=1, \ldots, k ; r=I_{\{j>i\}, \ldots \infty}$

where the function $I_{\{A\}}$ is 1 if condition $A$ is true and 0 otherwise. For an explanation of the number $r k+i-j$ in (14), see Figure 2.

$<$ Figure 2 about here $>$

Let the random variable $\hat{N}(i)_{L-t}$ denote the total number of arrivals in the time interval $[\tau-L, \tau-t)$ given that we are in phase $i$ at time point $\tau-t$. This has probability distribution

$$
P\left(\hat{N}(i)_{L-t}=u\right)=e^{-\lambda(L-t)} \sum_{v=\max \{u k+1-i, 0\}}^{(u+1) k-i} \frac{(\lambda(L-t))^{v}}{v !}
$$

Let the random variable $C_{L}(t \mid r, u)$ denote the aggregate committed demand (see the previous definition) in the time interval $[\tau-L, \tau)$ given that one has observed $r$ arrivals in the time interval $[\tau$ $t, \tau)$ and $u$ arrivals in the time interval $[\tau-L, \tau-t)$. This has probability distribution

$$
P\left(C_{L}(t \mid r, u)=x\right)=\sum_{y=0}^{\min \{r, x-u\}}\left(\begin{array}{l}
r \\
y
\end{array}\right)(P(X \leq q))^{y}(P(X>q))^{r-y} P\left(Q^{\operatorname{Reg}}(y)+Q(u)=x\right)
$$

Then the probability distribution of $\bar{C}_{L}(t)$ can be specified as follows

$$
P\left(\bar{C}_{L}(t)=x\right)=\sum_{i=1}^{k} \sum_{u=0}^{x} P\left(\hat{N}(i)_{L-t}=u\right) \sum_{r=0}^{\infty} F(i, r, t \mid 1) P\left(C_{L}(t \mid r, u)=x\right)
$$

When $\tilde{\tau}$ is a randomly chosen time point, we will at this time point be in phase is $j$ with probability $1 / k$. Therefore, the probability distribution of $\tilde{C}_{L}(t)$ can be specified as follows

$$
P\left(\tilde{C}_{L}(t)=x\right)=\frac{1}{k} \sum_{j=1}^{k} \sum_{i=1}^{k} \sum_{u=0}^{x} P\left(\hat{N}(i)_{L-t}=u\right) \sum_{r=0}^{\infty} F(i, r, t \mid j) P\left(C_{L}(t \mid r, u)=x\right)
$$


This accomplishes that we can get computable expressions for doing numerical analysis of Postpone $(q, t)$ in the case of a compound Erlang process.

\subsection{Implementation}

Some of the expressions presented so far can be further simplified when $k=1$, that is the demand process is a compound Poisson process, by using the recursion scheme of Adelson (1966). Actually, we have developed computer codes, programmed in Visual Basic for Excel, both for the general case ( $k$ any positive integer) and the case $k=1$, in order to validate our computer codes as well as possible. This is done for both rules $\operatorname{Split}(q)$ and Postpone $(q, t)$.

\subsection{The case $S \leq q$}

For this case in particular, the rule $\operatorname{Split}(q)$ is superfluous as not even the truncated suborder of a larger order has any chance of receiving full service. Note that Postone $(q, 0)$ represents the case where larger orders are not discriminated. We can prove

\section{Proposition 1}

When $S \leq q$, the performance measures (OFR and average on-hand inventory) are identical for the rules Postpone (q,0) and $\operatorname{Split}(q)$.

Proof: see Appendix.

\subsection{Choice of $t$}

We derive what we find is a reasonable threshold value of $t$, making customers of larger orders indifferent between Postpone $(q, t)$ and $\operatorname{Split}(q)$. Consider a large order whose size can be specified by the random variable $X^{\text {Lar }}$ which has probability distribution

$$
P\left(X^{L a r}=x\right)=\frac{P(X=x)}{P(X>q)} \quad x=q+1, q+2, \ldots
$$

In Postpone (q,t) a measure for accumulated waiting time of all units of a larger order is $E\left[X^{\text {Lar }}\right] t$. This measure is slightly optimistic as we here assume that any larger order is instantaneously served after $t$ time units of postponement. Similarly, in $\operatorname{Split}(q)$ a measure for the accumulated waiting time can be specified as $E\left[\max \left\{X^{L a r}-q, 0\right\}\right] L$. Also this measure is slightly optimistic as we here assume that the truncated suborder (of size $q$ ) is served instantaneously. Assuming that they are equally optimistic, the value of $t$ that equalizes these two terms is

$$
t=\frac{L \sum_{j=q+1}^{\infty}(j-q) P(X=j)}{\sum_{j=q+1}^{\infty} j P(X=j)}
$$


The right hand side of (20) belongs to the interval between 0 and $L$. We find that the right hand side of (20) is a good estimate of a $t$ value that makes customers of larger orders indifferent between Postpone $(q, t)$ and Split $(q)$. Developing exact expressions would be very difficult.

\section{Numerical results}

Throughout this section, we keep $L$ fixed at level 4 and we assume that the random variable $X$ is (delayed) geometrically distributed. This means that it has probability distribution

$$
P(X=j)=(1-\rho) \rho^{j-1} \quad j=1,2, \ldots
$$

where the parameter $\rho$ is in the interval between 0 and 1 . The use of the word "delayed" is due to Zipkin (2000, p. 451). Then (20) simplifies to

$$
t=\frac{1}{q+1-\rho q} L
$$

and the demand rate $d$ is given as

$$
d=\frac{\lambda}{k(1-\rho)}
$$

When $k=1$, this demand process is called a "stuttering" Poisson process (see Axsater (2006) p. 82). In Johnston et al. (2003), some empirical evidence is given for the relevance of such a demand model. First, we illustrate the impact of the parameter $t$ on the performance of $\operatorname{Postpone}(q, t)$. For the case where $d=2.5, \rho=0.8, q$ is the $90 \%$ quantile (that is, the least integer value of $x$ where $P(X \leq x)$ $\geq 0.9$ ) and the order fill rate $\beta=0.9$ (that is $S$ is chosen such that $O F R \geq \beta$ ), Figures 3 and 4 show how the average inventory level depends on the parameter $t$ when $k=1$ and 2 , respectively. The reason for the curves not being monotonically decreasing in $t$ is because $S$ is restricted to being an integer.

\section{$<$ Figures 3 and 4 about here $>$}

We now make a systematic evaluation of how the two rules compare to each other, assuming that $t$ given by (22) will make the customers of large orders indifferent between these. We construct a series of data sets in the following way: we let $q$ be either the $90 \%$ or the $95 \%$ quantile (see definition above) of $X$. The order fill rate $\beta$ is either 0.9 or 0.95 . We let the demand rate $d$ attain the values $1.2,2.5,3.75,4$ (these perhaps seem more obvious choices when multiplying by $L$ as $d L=5$, $10,15,20$ ). For each choice of $d$, we then let $\rho=0.5,0.6,0.7,0.8,0.9$. In order also to investigate the impact of deviating from the common Poisson process assumption, we let $k=1$ and 2 . The remaining parameter $\lambda$ is given by (23). Combining all these parameter values gives a total of 160 data sets. In the histograms in Figures 5 and 7, we have stated the reduction in inventory levels (measured in $\%$ ) when going from a situation with no discrimination (which is Postpone $(q, 0)$ ) to a case with Postpone $(q, t)$ (and $t$ given by (22)) for the cases of $k=1$ and $k=2$ when pooling all data 
together. Similarly, in the histograms in Figures 6 and 8, we have stated the percentage reduction in inventories when going from no discrimination to using rule Split(q).

$<$ Figures 5-8 about here $>$

When comparing Figure 5 to Figure 6 and Figure 7 to Figure 8, we see for both $k=1$ and $k=2$ that a higher inventory reduction is obtained by using $\operatorname{Split}(q)$ rather than using Postpone $(q, t)$. When $k=$ 1 , the average inventory reduction is $5.49 \%$ for $\operatorname{Postpone}(q, t)$ and it is $9.34 \%$ for $\operatorname{Split}(q)$ and in all the 80 cases the largest inventory reduction is achieved by $\operatorname{Split}(q)$. For $k=2$, the inventory reductions are: $3.78 \%$ for Postpone $(q, t), 10.95 \%$ for $\operatorname{Split}(q)$ and also here the largest inventory reduction is achieved by Split $(q)$ in all the 80 cases. As the dominance of Split $(q)$ over Postpone $(q, t)$ is more profound when $k=2$, the assumption about the demand process has significance for the conclusion. It emphasizes the importance of doing a careful analysis of the data of a demand process in order to specify the appropriate demand model. In a few cases, it turns out that the percentage reductions are negative. However, there is a logical explanation for this when considering Postpone ( $q, t)$, which can be realized by examining Figures 3 and 4, namely that if the base stock level $S$ does not decrease, the average inventory level will increase as $t$ is increased. Similarly for Split(q), when the optimal $S$ is unchanged, then naturally the average on-hand inventory in $\operatorname{Split}(q)$ will be larger than in the case with no discrimination. Also, in a few cases (occurring when $d$ is small and $\rho$ is high), we see that the computed $S$ is less than or equal to $q$, for which cases Proposition 1 holds.

\section{Concluding remarks}

Under a very general demand model, we have developed mathematical expressions for the average inventory level and order fill rate service measure (of the regular orders) of a base stock system when degraded service is enforced for customers of larger orders. The models (and corresponding computer codes) may be valuable tools for an organization to explore the impact of various strategies for degrading the service of larger orders; particularly in recognition of the fact that these orders may have a very disruptive effect upstream in the supply chain. Our numerical investigations reveal that in what we find is a fair comparison (by (22)), Split(q) seems to be a better choice than Postpone $(q, t)$. It also seems that deviating from the common Poisson process assumption does have some impact on this conclusion which justifies our choice of using a more general demand model for the investigation. Though we examine a fairly large number of data sets we look exclusively at the geometric distribution to describe order sizes. So obviously, further numerical studies could be devoted to examining other distributions, for instance the negative binomial, the Poisson or the logarithmic distribution. One could interpret Pospone $(q, t)$ not as a rule for degrading service but as a special case of an advanced-demand information system where all receipts of larger orders are known $t$ time units in advance. For studies of inventory systems in the presence of advanceddemand information, see among others Harihan and Zipkin (1995), Gallego and Özer (2001) and Marklund (2006). Therefore, the mathematical models developed in this paper could also be seen in a slightly different perspective, namely by studying aspects of advanced-demand information systems. Of course, the mathematical model can easily be adapted such that it need not be solely the customers of larger orders that provide advanced-demand information. 


\section{References}

Adelson RM (1966). Compound Poisson distributions. Operational Research Quarterly, 17: 73-75. Axsäter S (2006). Inventory Control, 2nd ed. Springer's International Series. Springer: New York.

Cohen MA, Zheng Y-S, Wang Y (1999). Identifying opportunities for improving Teradyne's service-parts logistics system. Interfaces, 29 (4): 1-18.

Cox DR (1962). Renewal Theory. Methuen: London.

Gallego G, Özer Ö (2001). Integrating replenishment decisions with advance demand information. Management Science, 47: 1344-1360.

Harihan R, Zipkin P (1995). Customer-order information, leadtimes and inventories. Management Science, 41: 1599-1607.

Johnston FR, Boylan JE and Shale EA (2003). An examination of the size of orders from customers, their characterization and the implication for inventory control of slow moving items. Journal of the Operational Research Society, 54: 833-837.

Kocaga YL, Sen A (2007). Spare parts inventory management with demand lead times and rationing. IIE Transactions, 39: 879-898.

Larsen C, Seiding CH, Teller C and Thorstenson A (2008). An inventory control project in a major Danish company using compound renewal demand models. IMA Journal of Management Mathematics, 19: 145-162.

Larsen C, Thorstenson A (2008). A comparison between the order and the volume fill rate for a base-stock inventory control system under a compound renewal demand process. Journal of the Operational Research Society, 59: 798-804.

Marklund J (2006). Controlling inventories in divergent supply chains with advance-order information. Operations Research, 54: 988-1010.

Teunter R, Haneveld WKK (2008). Dynamic inventory rationing strategies for inventory systems with two demand classes, Poisson demand and backordering. European Journal of Operational Research, 190: 156-178.

Thomas DJ, Tyworth JE (2006). Pooling lead-time risk by order splitting: a critical review. Transportation Research Part E, 42: 245-257.

Tijms HC (2003). A first course in stochastic models. John Wiley: Chichester.

Wang Y, Cohen MA and Zheng Y-S (2002). Differentiating customer service on the basis of delivery lead-times. IIE Transactions, 34: 979-989. 
Zipkin PH (2000). Foundations of inventory management. McGraw-Hill: Boston. 


\section{Appendix}

\section{Proof of Lemma 1}

We prove by induction in $m$. When $m=1$, the result is given from (1). Consider now an integer $m$ ' $\geq 1$ and assume that we have shown the result for $m=m^{\prime}-1$. For any $x=0,1, \ldots, q$, it holds that

$$
\begin{aligned}
& P\left(Q\left(m^{\prime}\right)=x\right)=\sum_{y=0}^{x} P\left(Q\left(m^{\prime}-1\right)=y\right) P(X=x-y)=(P(X \leq q))^{m^{\prime}} \sum_{y=0}^{x} P\left(Q^{\operatorname{Re} g}\left(m^{\prime}-1\right)=y\right) P\left(X^{\operatorname{Re} g}=x-y\right) \\
& =(P(X \leq q))^{m^{\prime}} P\left(Q^{\operatorname{Re} g}\left(m^{\prime}\right)=x\right)
\end{aligned}
$$

\section{Proof of Proposition 1}

We claim that for any $x=0,1, \ldots, q-1$, it holds that $P\left(\bar{D}_{L}=x\right)=P\left(\bar{C}_{L}(0)=x\right)$. First, we consider the random variable $\bar{C}_{L}(0)$. When $t=0$, the random variable $\bar{N}_{t}$ is identical to zero. This means that the summation on the right hand side of (10a) vanishes to zero. Also, the right hand side of (10b) only gives non-zero terms if index $r=0$. This again forces index $m$ to be les than or equal to $x$ and index $y$ to be zero. Therefore, we get

$$
P\left(\bar{C}_{L}(0)=x\right)=\left\{\begin{array}{cc}
P\left(N(\bar{\tau})_{L}=0\right) & x=0 \\
\sum_{m=1}^{x} P\left(N(\bar{\tau})_{L}=m\right) P(Q(m)=x) & x=1,2, \ldots
\end{array}\right.
$$

Now we consider the random variable $\bar{D}_{L}$. If $q=1$, our claim follows by comparing (A1) to (6.a). Assume $q>1$. When $x \leq q-1$, the inequality $(q m-x) /(q-1)>m-1$ is equivalent to the inequality $q+$ $m>x+1$, which is true when $q>x$, implying $\operatorname{Roof}((q m-x) /(q-1))=m$ (note $(q m-x) /(q-1)$ is always less than or equal to $m$ ). When $q>x$, it also holds that $\operatorname{Roof}(x / q)=1$. Therefore, we get from (6a-b) that

$$
P\left(\bar{D}_{L}=x\right)=\left\{\begin{array}{cc}
P\left(N(\bar{\tau})_{L}=0\right) & x=0 \\
\sum_{m=1}^{x} P\left(N(\bar{\tau})_{L}=m\right)(P(X \leq q))^{m} P\left(Q^{\operatorname{Re} g}(m)=x\right) & x=1,2, \ldots, q-1
\end{array}\right.
$$

Lemma 1 now verifies our claim. Similarly, we can prove that for any $x=0,1, . ., q-1$, it holds that $P\left(\tilde{D}_{L}=x\right)=P\left(\tilde{C}_{L}(0)=x\right)$. As it is the probabilities $P\left(\bar{D}_{L}=x\right)=P\left(\bar{C}_{L}(0)=x\right)$ for $x=0,1, \ldots, S-1$ that specify the $O F R$ expressions in (5) and (11) and it is the probabilities $P\left(\tilde{D}_{L}=x\right)=P\left(\tilde{C}_{L}(0)=x\right)$ for $x=0,1, \ldots, S-1$ that specify the average on-hand inventory expressions in (7) and (13), the result follows from $S \leq q-1$. 
Figures

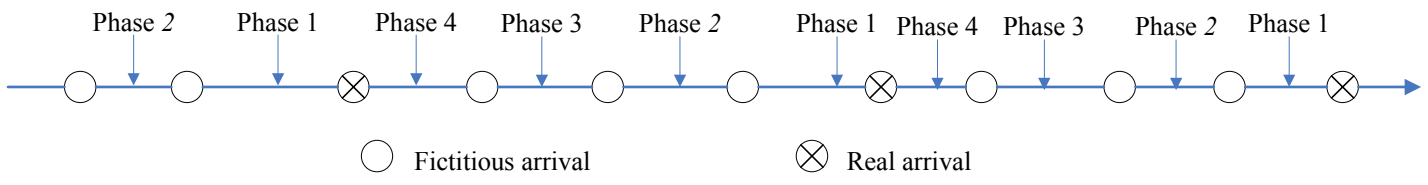

Figure 1: Illustration of an Erlang process with $k=4$.

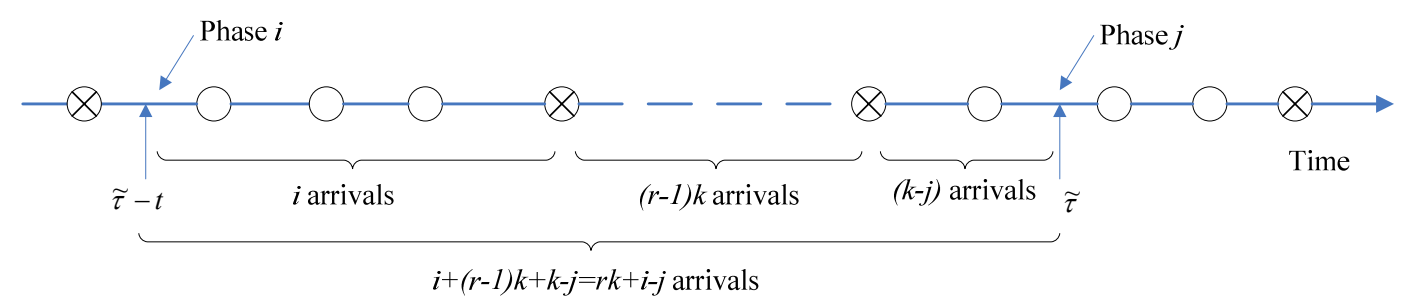

Figure 2: Explanation for (14). 


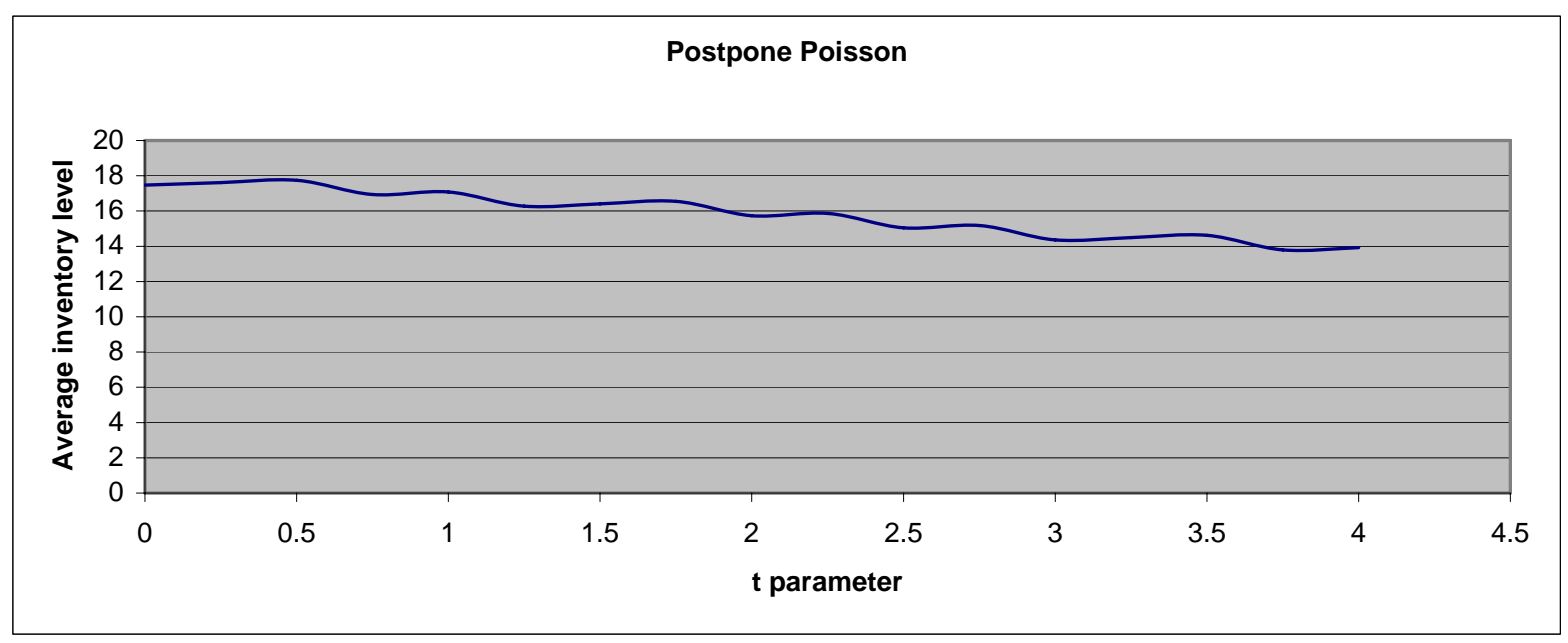

Figure 3: Postpone (q, $t$ ) with $k=1, \rho=0.8, \lambda=0.5, q$ is the $90 \%$ quantile, $\beta=0.9$. The corresponding value for Split is 16.09. The curve is interpolated through the computed values for $t$ $=0,0.25,0.5, \ldots, 3.75,4$.

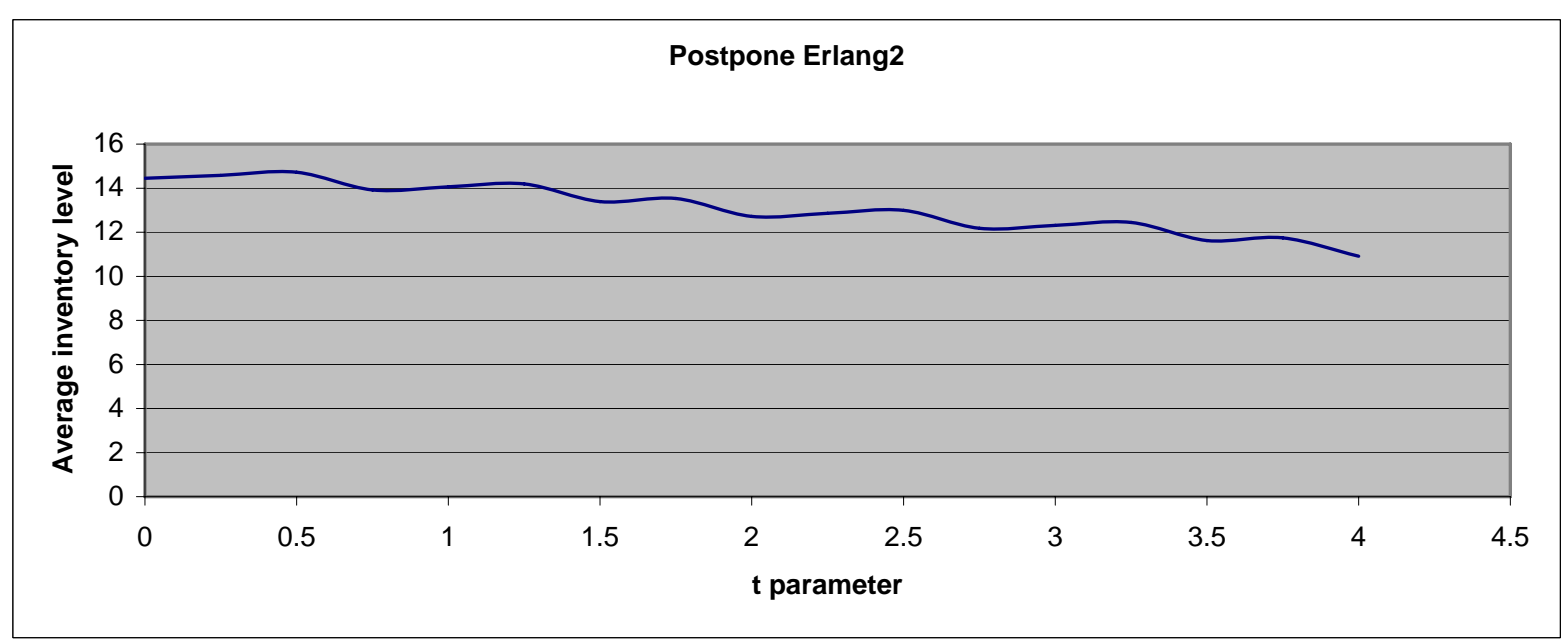

Figure 4: Postpone (q, $t$ ) with $k=2, \rho=0.8, \lambda=1, q$ is the $90 \%$ quantile, $\beta=0.9$. The corresponding value for Split is 12.11. For further comments, see Figure 3. 


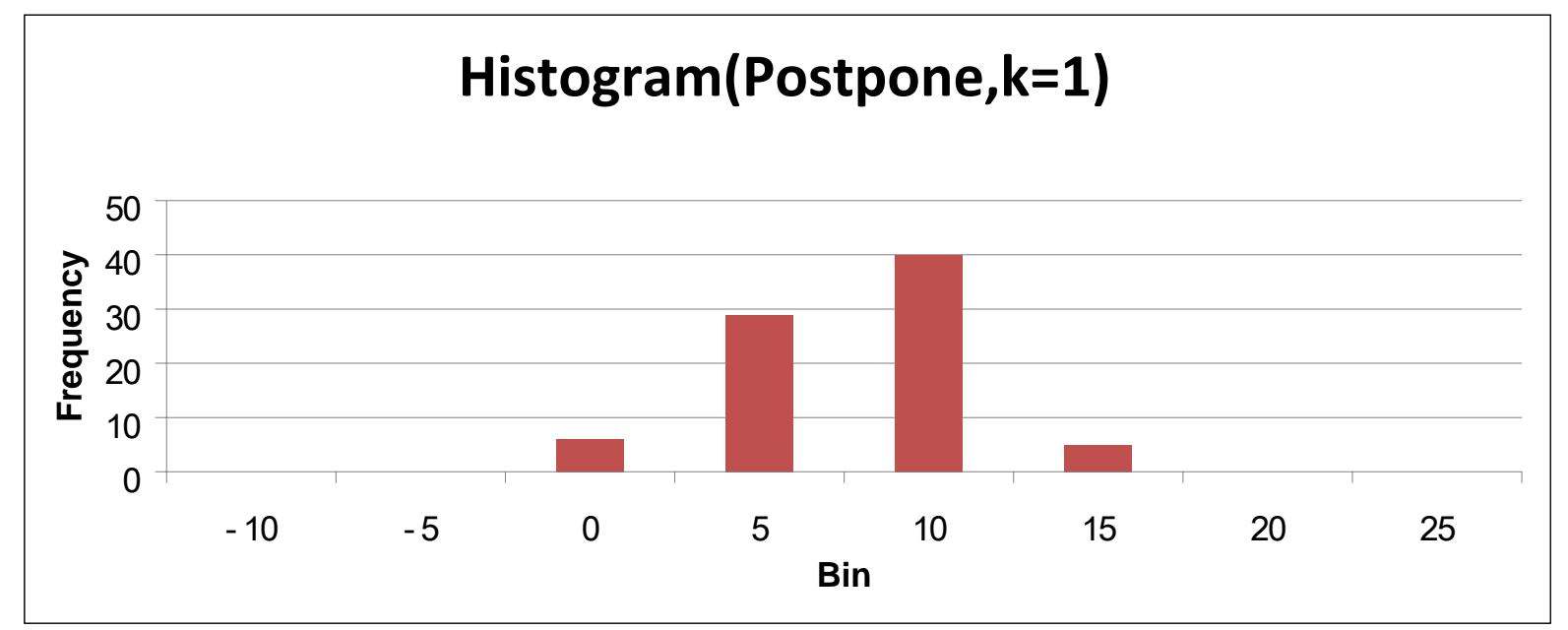

Figure 5: Percentage reductions in the inventory level for all results of Postpone $(q, t)$ with $t$ given by (22) and $k=1$. All observations in Bin $=0$ are in the interval $[-5,0)$ and so forth. There is a total of 80 observations.

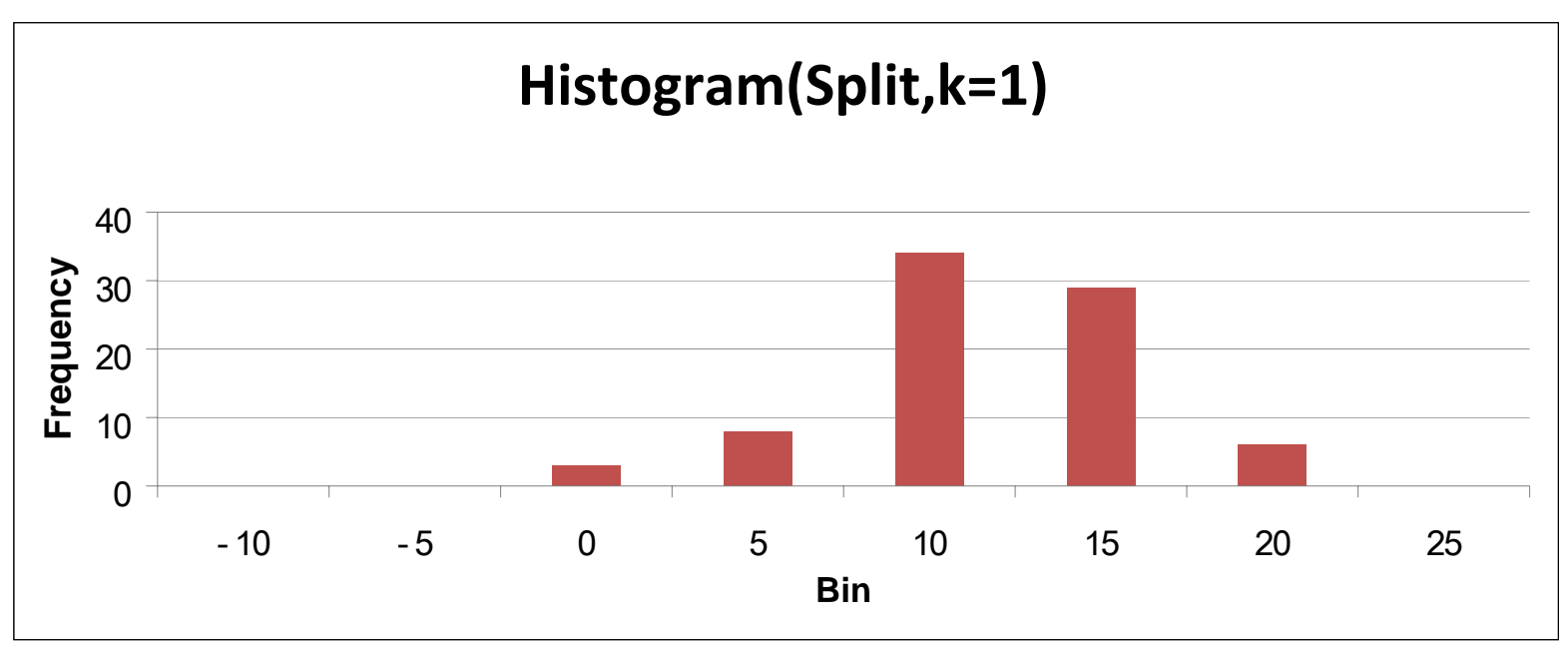

Figure 6: Percentage reductions in the inventory level for all results of $\operatorname{Split}(q)$ with $k=1$. For further comments, see Figure 5. 


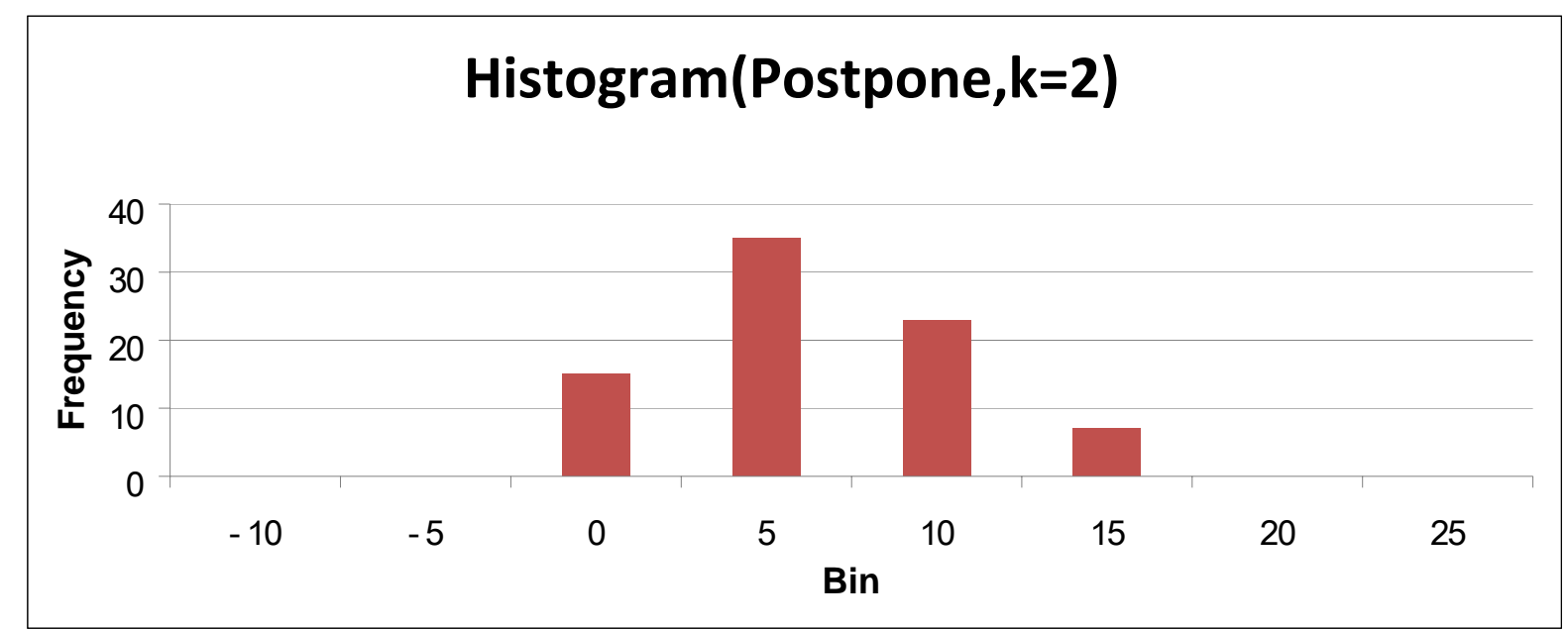

Figure 7: Percentage reductions in the inventory level for all results of Postpone $(q, t)$ with $t$ given by (22) and $k=2$. For further comments, see Figure 5.

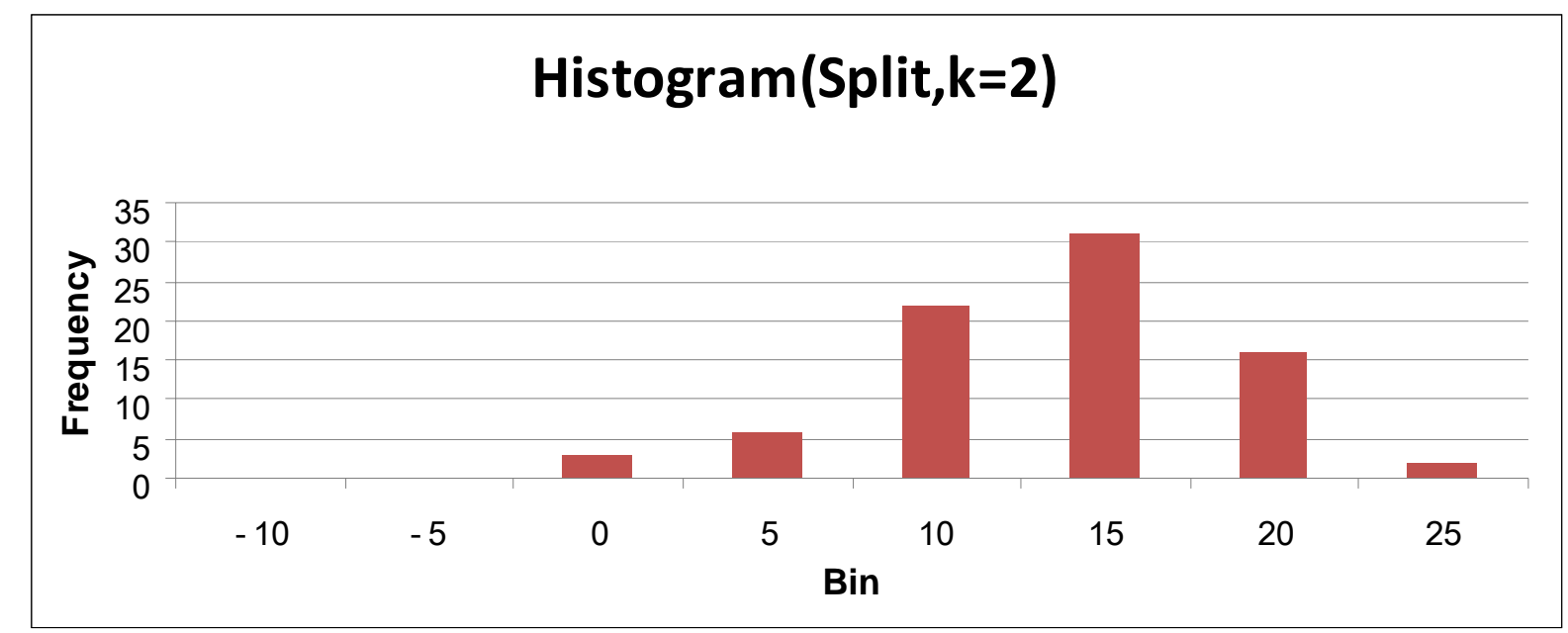

Figure 8: Percentage reductions in the inventory level for all results of $\operatorname{Split}(q)$ with $k=2$. For further comments, see Figure 5. 


\section{Working Papers from CORAL - Centre for Operations Research Applications in Logistics}

L-2008-07 Bisheng Du \& Christian Larsen: Base stock policies with degraded service to larger orders.

L-2008-06 Karina H. Kjeldsen: Classification of routing and scheduling problems in liner shipping.

L-2008-05 Daniele Pretolani, Lars Relund Nielsen, Kim Allan Andersen \& Matthias Ehrgott: Time-adaptive versus history-adaptive strategies for multicriterion routing in stochastic time-dependent networks.

L-2008-04 Christian Larsen: Comments to Özkaya et al. (2006).

L-2008-03 Christian Larsen: Derivation of confidence intervals of service measures in a base-stock inventory control system with low-frequent demand.

L-2008-02 Jens Lysgaard: The Pyramidal Capacitated Vehicle Routing Problem.

L-2008-01 Jens Lysgaard \& Janni Løber: Scheduling participants of Assessment Centres.

L-2007-03 Christian Larsen: Note: Comments on the paper by Rosling (2002).

L-2007-02 Christian Larsen, Claus Hoe Seiding, Christian Teller \& Anders Thorstenson: An inventory control project in a major Danish company using compound renewal demand models.

L-2007-01 Christian Larsen: The $Q(s, S)$ control policy for the joint replenishment problem extended to the case of correlation among item-demands.

L-2006-11 Daniele Pretolani, Lars Relund Nielsen \& Kim Allan Andersen: A note on "Multicriteria adaptive paths in stochastic, time-varying networks".

L-2006-10 Lars Relund Nielsen, Kim Allan Andersen \& Daniele Pretolani: Bicriterion a priori route choice in stochastic time-dependent networks.

L-2006-09 Christian Larsen \& Gudrun P. Kiesmüller: Developing a closed-form cost expression for an $(R, s, n Q)$ policy where the demand process is compound generalized Erlang.

L-2006-08 Eduardo Uchoa, Ricardo Fukasawa, Jens Lysgaard, Artur Pessoa, Marcus Poggi de Aragão, Diogo Andrade: Robust Branch-Cut-and-Price for the Capacitated Minimum Spanning Tree Problem over a Large Extended Formulation.

L-2006-07 Geir Brønmo, Bjørn Nygreen \& Jens Lysgaard: Column generation approaches to ship scheduling with flexible cargo sizes. 
L-2006-06 Adam N. Letchford, Jens Lysgaard \& Richard W. Eglese: A Branch-andCut Algorithm for the Capacitated Open Vehicle Routing Problem.

L-2006-05 Ole Mortensen \& Olga W. Lemoine: Business integration between manufacturing and transport-logistics firms.

L-2006-04 Christian H. Christiansen \& Jens Lysgaard: A column generation approach to the capacitated vehicle routing problem with stochastic demands.

L-2006-03 Christian Larsen: Computation of order and volume fill rates for a base stock inventory control system with heterogeneous demand to investigate which customer class gets the best service.

L-2006-02 Søren Glud Johansen \& Anders Thorstenson: Note: Optimal base-stock policy for the inventory system with periodic review, backorders and sequential lead times.

L-2006-01 Christian Larsen \& Anders Thorstenson: A comparison between the order and the volume fill rates for a base-stock inventory control system under a compound renewal demand process.

L-2005-02 Michael M. Sørensen: Polyhedral computations for the simple graph partitioning problem.

L-2005-01 Ole Mortensen: Transportkoncepter og IT-støtte: et undersøgelsesoplæg og nogle foreløbige resultater.

L-2004-05 Lars Relund Nielsen, Daniele Pretolani \& Kim Allan Andersen: $K$ shortest paths in stochastic time-dependent networks.

L-2004-04 Lars Relund Nielsen, Daniele Pretolani \& Kim Allan Andersen: Finding the $K$ shortest hyperpaths using reoptimization.

L-2004-03 Søren Glud Johansen \& Anders Thorstenson: The $(r, q)$ policy for the lostsales inventory system when more than one order may be outstanding.

L-2004-02 Erland Hejn Nielsen: Streams of events and performance of queuing systems: The basic anatomy of arrival/departure processes, when focus is set on autocorrelation.

L-2004-01 Jens Lysgaard: Reachability cuts for the vehicle routing problem with time windows. 
ISBN 9788778823588

Department of Business Studies

Aarhus School of Business

University of Aarhus

Fuglesangs Allé 4

DK-8210 Aarhus V - Denmark

Tel. +4589486688

Fax +4586150188

www.asb.dk 